\title{
Inhibition of vasculogenesis, but not angiogenesis, prevents the recurrence of glioblastoma after irradiation in mice
}

\author{
Mitomu Kioi, ${ }^{1}$ Hannes Vogel, ${ }^{2}$ Geoffrey Schultz, ${ }^{3}$ Robert M. Hoffman,4,5 \\ Griffith R. Harsh, ${ }^{6}$ and J. Martin Brown ${ }^{1}$

\begin{abstract}
${ }^{1}$ Division of Radiation and Cancer Biology, Department of Radiation Oncology, ${ }^{2}$ Department of Pathology, and ${ }^{3}$ Department of Surgery, Stanford University School of Medicine, California. ${ }^{4}$ AntiCancer Inc., San Diego, California. ${ }^{5}$ Department of Surgery, University of California, San Diego.
\end{abstract} \\ ${ }^{6}$ Department of Neurosurgery, Stanford University School of Medicine.
}

\begin{abstract}
Despite the high doses of radiation delivered in the treatment of patients with glioblastoma multiforme (GBM), the tumors invariably recur within the irradiation field, resulting in a low cure rate. Understanding the mechanism of such recurrence is therefore important. Here we have shown in an intracranial GBM xenograft model that irradiation induces recruitment of bone marrow-derived cells (BMDCs) into the tumors, restoring the radiation-damaged vasculature by vasculogenesis and thereby allowing the growth of surviving tumor cells. BMDC influx was initiated by induction of HIF-1 in the irradiated tumors, and blocking this influx prevented tumor recurrence. Previous studies have indicated that BMDCs are recruited to tumors in part through the interaction between the HIF-1-dependent stromal cell-derived factor-1 (SDF-1) and its receptor, CXCR4. Pharmacologic inhibition of HIF-1 or of the SDF-1/CXCR4 interaction prevented the influx of BMDCs, primarily CD11 $\mathrm{b}^{+}$myelomonocytes, and the postirradiation development of functional tumor vasculature, resulting in abrogation of tumor regrowth. Similar results were found using neutralizing antibodies against CXCR4. Our data therefore suggest a novel approach for the treatment of GBM: in addition to radiotherapy, the vasculogenesis pathway needs to be blocked, and this can be accomplished using the clinically approved drug AMD3100, a small molecule inhibitor of SDF-1/CXCR4 interactions.
\end{abstract}

\section{Introduction}

Radiotherapy plays a major role in the treatment of glioblastoma multiforme (GBM). However, despite the use of radiosensitizers, radioactive implants, the combination of radiation with anticancer drugs, and most recently, high-dose boost stereotactic radiosurgery (1), GBMs invariably recur, leading to the death of more that $75 \%$ of the patients by 2 years. Importantly, most of the recurrences occur within the radiation field (2-4). Thus, any method of increasing the local control of the primary tumor by radiotherapy would produce a major improvement in the curability of GBM. At first sight, it is difficult to understand how the large radiation doses given locally to brain tumors do not result in cures, because even though all of the tumor cells may not be sterilized by radiation, it is unlikely that any of the endothelial cells in the tumor could survive the large doses given. Indeed, recent data demonstrate that the response of tumors to irradiation can be affected by the sensitivity of tumor endothelial cells $(5,6)$.

The tumor vasculature can arise from sprouting and proliferation of endothelial cells from local vessels (angiogenesis), co-option of preexisting vessels, or by colonization of circulating endothelial or other cells primarily from the BM (a phenomenon known as vasculogenesis). The paradox of the sensitivity of the vasculature to irradiation and the resistance of GBMs to radiotherapy could be resolved if, as postulated, circulating cells outside of the radiation field can recolonize and/or stabilize the tumor vasculature after irradiation, thereby supporting any remaining viable tumor cells. This resto-

Conflict of interest: Robert M. Hoffman and J. Martin Brown have significant equity holdings in AntiCancer Inc. and Proacta Inc., respectively.

Citation for this article: J Clin Invest. 2010;120(3):694-705. doi:10.1172/JCI40283. ration of the vasculature facilitated by bone marrow-derived cells (BMDCs) is observed in ischemic normal tissues and in malignant tissues (7-10). These BMDCs are also thought to create an environment for tumor growth and invasion, and metastasis (11-13).

Among the variety of BMDCs contributing to vasculogenesis, the population most investigated is circulating endothelial progenitor cells (EPCs) $(8,10,14,15)$, which are thought to incorporate into the vascular endothelium. However, the extent of BMderived EPCs varies greatly with tumor type from 50\% (5) to very few (16). CD $45^{+}$monocytic lineage, including $\mathrm{CD} 11 \mathrm{~b}^{+}$myeloid cells, Tie $2^{+}$monocytes, VEGFR $1^{+}$hemangiocytes, and tumor-associated macrophages, has also been observed to incorporate into tumors. BM-derived myelomonocytic cells are often observed in the perivascular regions of the endothelium $(17,18)$ or colocalized with endothelial cells (19-21) and have been shown to stabilize the tumor vasculature (15).

In our previous study, we showed that mouse MT1A2 tumors subcutaneously implanted in preirradiated normal tissues produce a massive influx of BM-derived, MMP-9-expressing CD11 b ${ }^{+}$myelomonocytic cells into the tumors (22). Moreover, tumors cannot grow in preirradiated sites if the function of these monocytes is abrogated by growing the tumors in MMP-9-knockout mice. We postulated that this is a result of the fact that the local irradiation abrogates the sprouting and proliferation of endothelial cells from adjacent normal vessels, thereby forcing the tumor to rely on the "backup" pathway of vasculogenesis from circulating endothelial and other proangiogenic cells. In such a situation, blocking the vasculogenesis pathway (by deleting MMP-9) prevented tumor growth.

In the present study, we have tested the hypothesis that blocking the vasculogenesis pathway could improve tumor control by 
irradiation using an orthotopic model of GBM. We have identified 3 key components of the vasculogenesis pathway involving the mobilization of proangiogenic BMDCs and their recruitment to tumors. First, solid tumors develop regions of hypoxia, leading to an induction of the transcription factor HIF-1 (23), which has been shown to be a major player in the recruitment of BMDCs to tumors including GBM (24) and to ischemic regions of normal tissues (7). Importantly, tumors regrowing after irradiation or transplanted into an irradiated tissue are more hypoxic than control tumors (25-27) and would therefore be expected to have higher levels of HIF-1. In addition, there is evidence that local tumor irradiation and reoxygenation can induce HIF-1 in tumors (28). Thus, we postulated that inhibition of HIF-1 after tumor irradiation would reduce or abrogate the accumulation of proangiogenic BMDCs into tumors. Second, several investigators have shown that BMDCs are recruited to and retained in hypoxic normal tissues or in tumors by the hypoxia-dependent secretion of stromal cell derived factor-1 (SDF-1, CXCL12) which binds its receptor, CXCR4, on the BMDCs $(7,29-32)$. Thus, we tested AMD3100, an inhibitor of the SDF-1/CXCR4 interaction (33), and neutralizing antibodies to CXCR4 in combination with local tumor irradiation. Finally, we tested the effect of monocyte/macrophage depletion on the ability of the GBM model tumor to regrow after irradiation. With all 3 of the above strategies to interfere with the vasculogenesis pathway, we show that the radiation-induced uptake of BMDCs, specifically CD $11 \mathrm{~b}^{+}$myelomonocytes, into tumors can be prevented and that tumors can be controlled by doses of radiation that, in the absence of these strategies, are ineffective in sterilizing the tumors. Our data therefore suggest that inhibiting the vasculogenesis pathway after local tumor irradiation represents a new strategy that has the potential to increase the cure rate of human tumors by radiotherapy.

\section{Results}

Irradiation promotes recruitment of BMDCs into GBM. To investigate our hypothesis, we established an orthotopic brain tumor in a mouse model using human U251 GBM cells that were retrovirally transduced with the firefly luciferase gene in order to monitor tumor growth in real time. As shown in Figure 1, A and B, intracranially (i.c.) implanted U251 tumors grew progressively, and 5 daily doses of 2 or 4 Gy delivered locally to the tumors by irradiating the whole brain caused the tumors to regress and then regrow in a dose-dependent manner. We confirmed the tumor growth, infiltration into the surrounding normal brain, and regrowth after irradiation by histology and by MRI (Supplemental Figure 1, $\mathrm{A}$ and $\mathrm{B}$; supplemental material available online with this article; doi:10.1172/JCI40283DS1).

We next examined whether local irradiation induced the recruitment of BMDCs into the tumors. Tumor-bearing nude mice that had been transplanted earlier with GFP-expressing BM (34) were sacrificed when the tumors grew back to their preirradiation size after irradiation at either 8 or $15 \mathrm{~Gy}$. We found by both immunohistochemistry (IHC) and quantitative real-time PCR (qPCR) that irradiation induced BMDC influx in a dose-dependent manner (Figure 1, C and D, and Supplemental Figure 2A). If the cells that colonized the irradiated tumor were circulating EPCs derived from the BM, we would expect that the proportion of endothelial cells arising from the BM would be increased in tumors regrowing after radiation. However, we found that although the GFP BMDCs in the tumors were often associated with CD31-staining endothelial cells, they were not colocalized ( $<1 \%$, Supplemental Figure $2 B)$. This is in agreement with our previous study (22) that showed no increase in EPCs in tumors growing in preirradiated sites and is also consistent with several recent reports $(16,35-37)$. Significantly, we found that most of the induced influx of BMDCs into the tumors were $\mathrm{CD} 11 \mathrm{~b}^{+}$myelomonocytes (Figure 1, E and F). However, nonGFP CD $11 b^{+}$cells were modestly increased after irradiation (from 3 to $10 \mathrm{GFP}^{-} \mathrm{CD} 11 \mathrm{~b}^{+}$cells compared with 6 to $72 \mathrm{GFP}^{+} \mathrm{CD} 11 \mathrm{~b}^{+}$cells

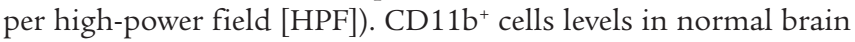
were very low compared with those in the irradiated tumor (Supplemental Figure 2C). Further characterization of the $\mathrm{CD}_{11 \mathrm{~b}^{+}}$cells in the irradiated tumors revealed that most of them did not express VEGFR 1 and VEGFR2. Approximately $20 \%$ of the CD $11 b^{+}$cells expressed $\mathrm{Gr}-1$. Interestingly, approximately $50 \%$ of the $\mathrm{CD}_{11} \mathrm{~b}^{+}$cells were Tie 2 positive, although they were not found in nonirradiated tumors (Figure 1G and Supplemental Figure 2D).

Irradiation causes vascular damage and increased tumor bypoxia. We postulated that the $\mathrm{CD} 11 \mathrm{~b}^{+} \mathrm{BMDC}$ recruitment is a result of the fact that local tumor irradiation sterilizes sufficient numbers of endothelial cells in and adjacent to the tumor to abrogate the sprouting and proliferation of endothelial cells from adjacent normal tissues and in tumor vessels, thereby forcing the tumor to rely on the vasculogenesis pathway stimulated by increased tumor hypoxia $(7,22,24)$. To test this, we determined the level of tumor endothelial cells, blood flow, and hypoxia by measuring CD31 expression, lectin perfusion, and pimonidazole staining after local tumor irradiation. We found that CD31-stained cells were reduced to some $25 \%$ of control levels by 2 weeks after 15 Gy of irradiation (Supplemental Figure 2E) and that this was accompanied by a large decrease in tumor perfusion and a large increase in tumor hypoxia (Figure 2, A and B). These data suggest that the increased tumor hypoxia caused by the severe vascular damage and decreased vessel perfusion after tumor irradiation could be the stimulus leading to the recruitment of BMDCs.

HIF-1 is a key factor responsible for BMDC influx by radiation and tumor recurrence. Consistent with our results (Figure 2, A and B), other investigators have shown that tumors recurring following irradiation or growing in an irradiated normal tissue are more hypoxic (25, 26,38 ) and would therefore be expected to have increased HIF-1 levels. To determine HIF-1 activity in real time in our brain-implanted U251 GBM, we stably expressed the HIF-1 reporter construct 5HRE-Luc (39) in U251 cells and monitored luciferase activity in control and irradiated tumors. By comparing the bioluminescence from the tumors expressing the constitutive pFB-Luc (proportional to total cells) with that of those expressing 5HRE-Luc (proportional to total HIF-1 activity), we could monitor tumor HIF-1 activity as a function of tumor size. HIF-1 levels were relatively high immediately after inoculation and then decreased, most likely as tumor vessels formed (data not shown). After that, HIF-1 levels increased as the tumor grew. As shown in Figure 2C, HIF-1 activity paralleled tumor growth in nonirradiated tumors but increased more rapidly than tumor growth starting at about 2 weeks after 15 Gy irradiation, indicating increased HIF-1 levels coincident with the increase in tumor hypoxia at this time.

To test our hypothesis that the increased HIF-1 levels were responsible for the increased influx of $\mathrm{CD} 11 \mathrm{~b}^{+}$cells into the tumors, we used the HIF-1 inhibitor NSC-134754 (40). This compound prevented the increased HIF-1 activity produced by hypoxia in vitro (data not shown) and by tumor irradiation in vivo (Figure 2D). When this inhibitor was given daily for 2 weeks, starting immediately after irradiation, 
A

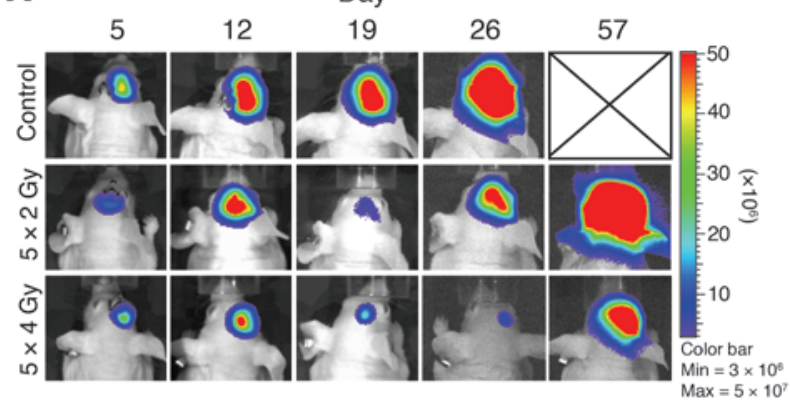

C

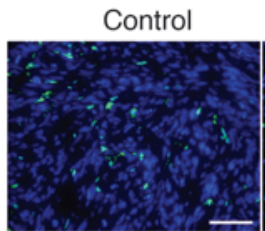

DAPI GFP

E

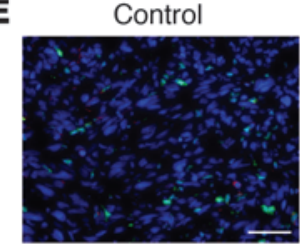

DAPI GFP CD11b

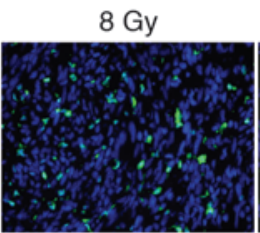

DAPI GFP

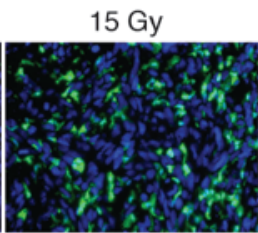

DAPI GFP

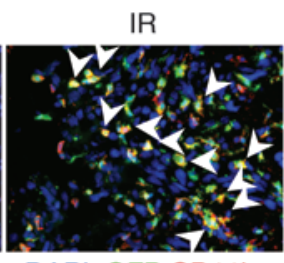

DAPI GFP CD11b

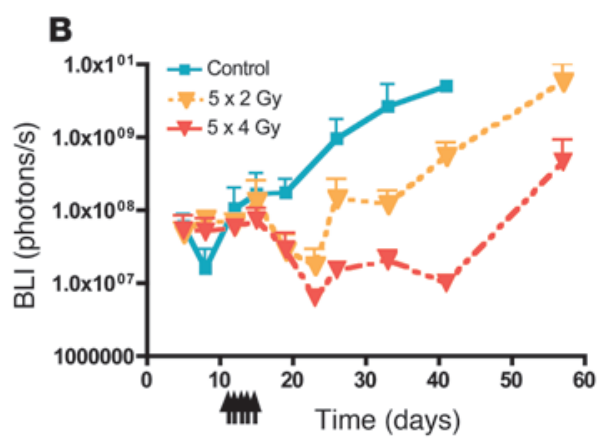

D 100
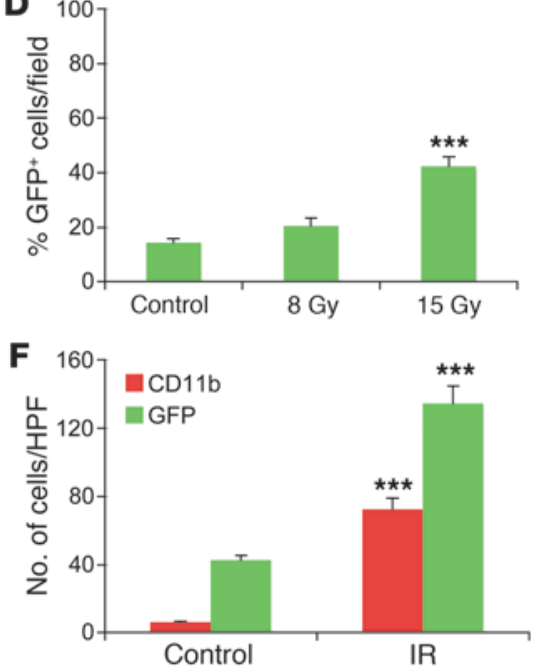

G

GAPI CD11b VEGFR1
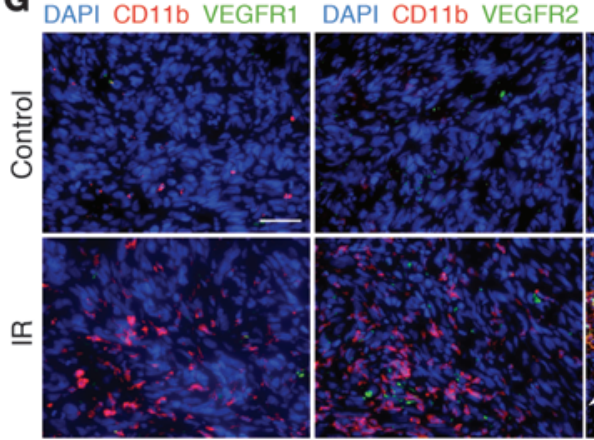

DAPI CD11b Tie-2

DAPI CD11b Gr-1
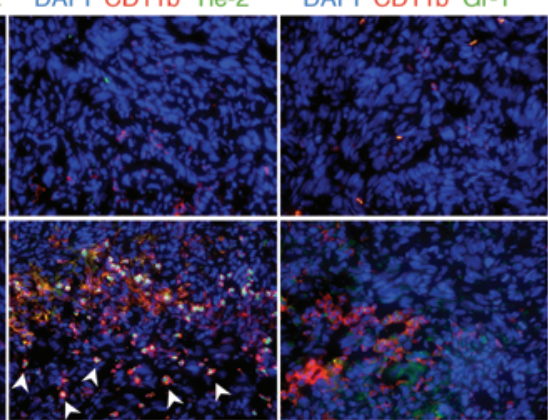

\section{Figure 1}

Irradiation promotes homing of BM-derived CD11 b+ myeloid monocytic cells into GBM. (A) Representative bioluminescent images of mouse heads with or without fractionated irradiation. Firefly luciferase-transduced U251 (U251/pFB-Luc) tumors were implanted i.c. and were irradiated from day 11 to day $15.5 \times 2$ Gy, 2 Gy irradiation 5 times daily. (B) Tumor radioresponse was measured by BLI ( $n=5$ per group). Error bars indicate SD. (C) Radiation induced the influx of BMDCs into the U251 i.c. tumor. GFP-BM-transplanted nude mice received whole brain irradiation at 0 , 8 , or 15 Gy on day 22. Scale bar: $50 \mu \mathrm{m}$. (D) Quantification of GFP-BM influx into the tumor. Error bars indicate SEM. ${ }^{\star \star \star} P<0.001$ versus control. (E) Representative images of IHC for GFP-BM cells and CD11 b+ myeloid monocytic cells in U251 i.c. tumor before and after irradiation. Arrowheads indicate CD11b+ GFP-BM cells. Scale bar: $50 \mu \mathrm{m}$. (F) Quantification of CD11b and GFP-BM influx into the tumor. Error bars indicate SEM. ${ }^{* * *} P<0.001$. (G) Characterization of CD11 b+ infiltrating cells into tumor after irradiation. Representative images of IHC staining for DAPI (blue), CD11b (red), and other markers (VEGFR1, VEGFR2, Tie-2, or Gr-1; green) in i.c. tumors after irradiation. Scale bar: $50 \mu \mathrm{m}$. IR, irradiation.

the increased tumor levels of $\mathrm{CD} 11 \mathrm{~b}^{+}$monocytes (and also the more mature macrophages, staining for F4/80) observed at 17 days after 15 Gy were abrogated (Figure 2, E and F). As shown in Supplemental Figure 3 , A and B, approximately $75 \%$ of infiltrating $C D 11 b^{+}$cells also expressed F4/80. These data indicate that HIF-1 plays a major role in the recruitment of BMDCs into tumors after radiotherapy.
To test our hypothesis that the recruitment into the tumor of $\mathrm{CD}_{11} \mathrm{~b}^{+}$and other proangiogenic cells promoted tumor growth after irradiation, we treated the mice with NSC-134754 in combination with local tumor irradiation. We started the drug delivery after irradiation to avoid any drug-induced changes in oxygen levels that would affect tumor radiosensitivity. Although 
A

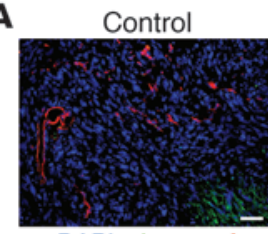

DAPI pimo perf

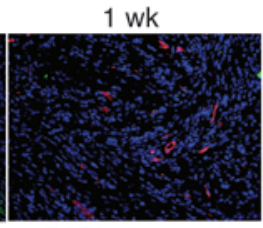

DAPI pimo perf

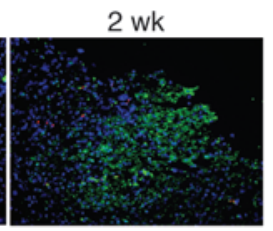

DAPI pimo perf

C

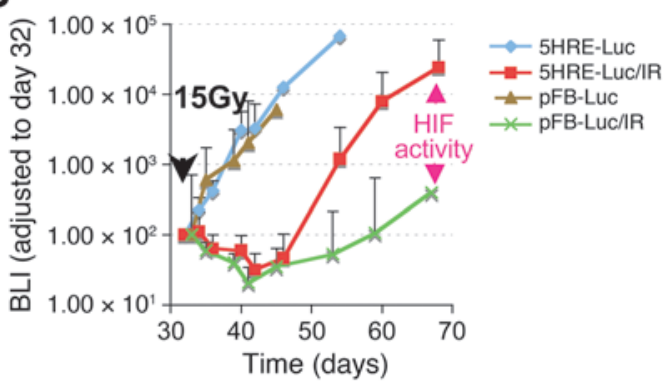

E

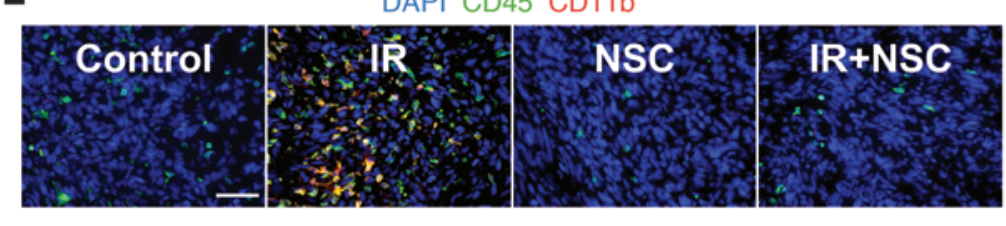

DAPI CD45 F4/80
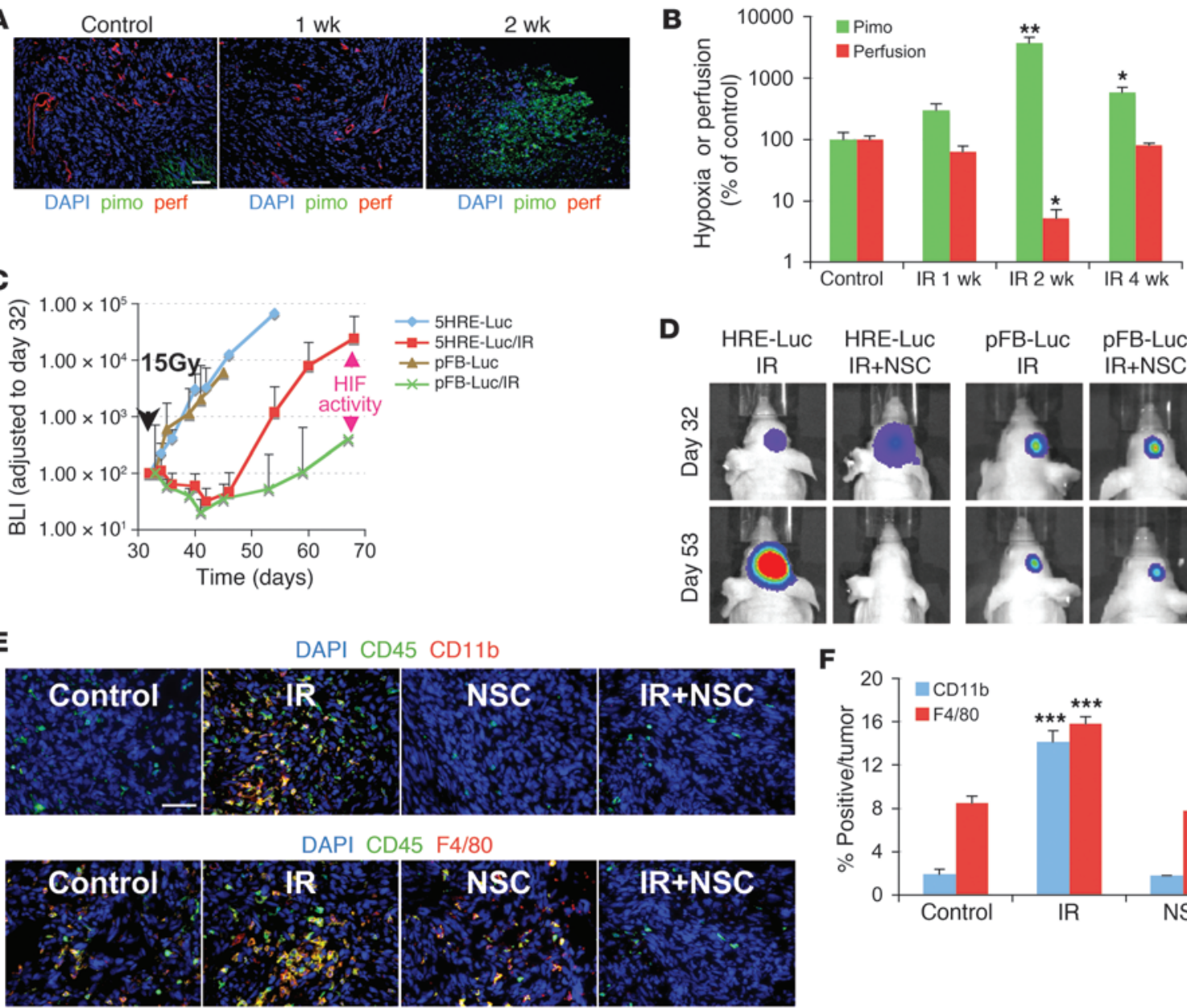

D

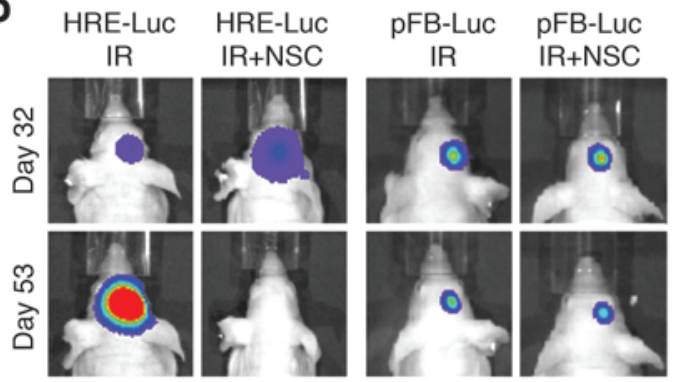

$\mathbf{F}$
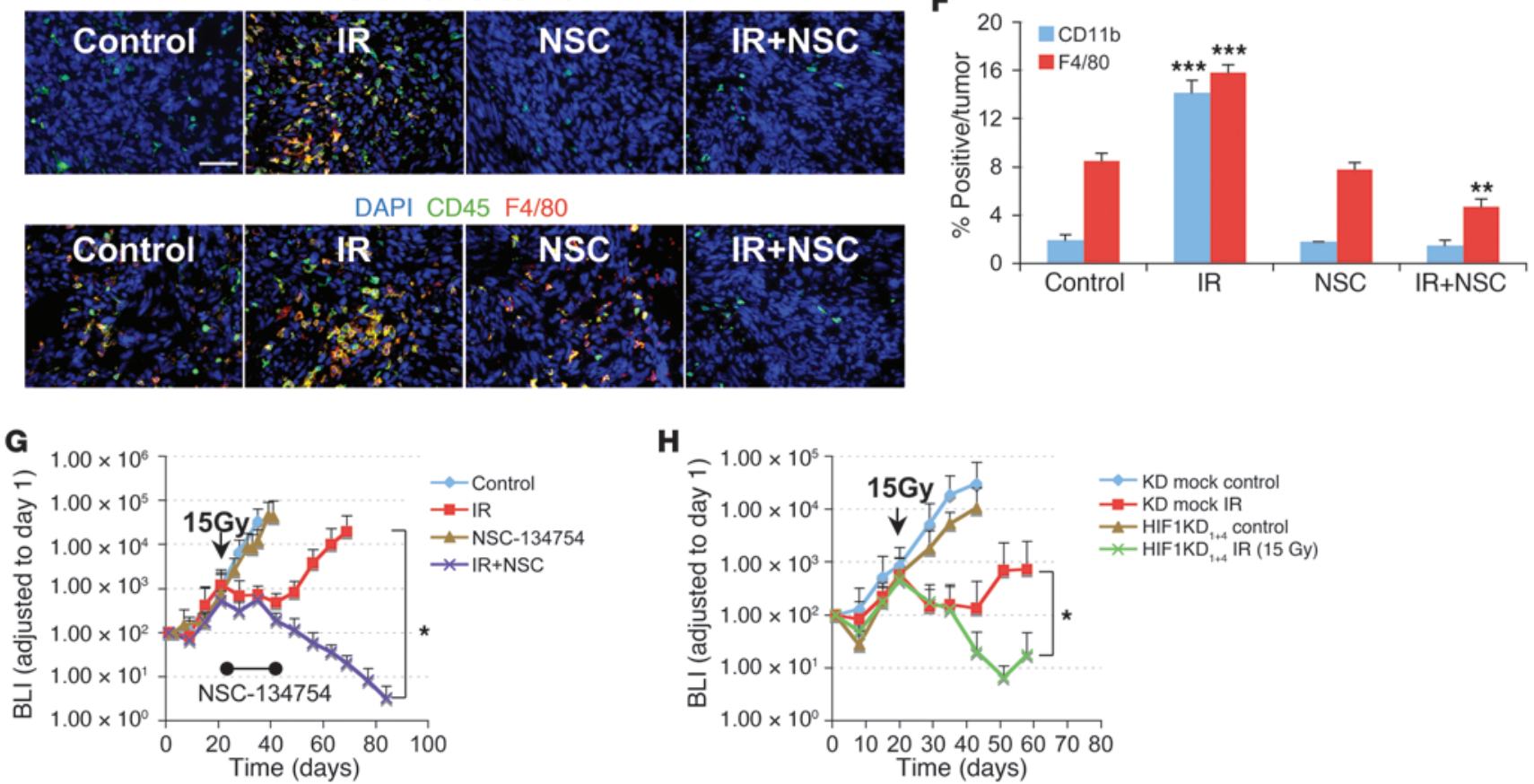

\section{Figure 2}

HIF-1 activity is increased in irradiated tumors and is necessary for influx of BMDCs and tumor recurrence after irradiation. (A) Representative images of IHC staining for hypoxia (pimonidazole [pimo]) and functional vessels (lectin perfusion [perf]) in i.c. tumors after irradiation. Scale bar: $50 \mu \mathrm{m}$. (B) Quantification of hypoxic areas and vessels in the tumors of A. Error bars indicate SEM. ${ }^{*} P<0.05$, ${ }^{* *} P<0.01$ versus control. Note the log scale on the ordinate. (C) BLI of 5HRE-Luc or pFB-Luc U251 i.c. tumors after a single dose (arrowhead) of irradiation on day 32 . The divergence of the 2 curves starting at about 2 weeks after irradiation indicates increased HIF-1 activity. Error bars indicate SD. (D) Effect of NSC134754 on inhibition of HIF-1 activity in vivo. 5HRE-Luc or pFB-Luc U251 tumor-bearing mice were treated with 15 Gy of irradiation, followed by daily injection of NSC-134754 for 3 weeks. Images were taken on the day of irradiation and 3 weeks later. (E) IHC staining for leukocyte (CD45) and monocyte (CD11b, top row) or macrophage ( $F 4 / 80$, bottom row) infiltration into i.c. tumors. Tumors were harvested on the day of irradiation for controls and 17 days after irradiation in treatment groups. Scale bar: $50 \mu \mathrm{m}$. (F) Quantification of CD11 b+ and F4/80+ cell influx in tumors of E. Error bars indicate SEM. ${ }^{* *} P<0.01,{ }^{* * *} P<0.001$ versus control. (G) Growth curves of i.c. tumors treated with irradiation, NSC-134754, irradiation+NSC-134754, and control ( $n=5$ per group). Error bars indicate SD. ${ }^{*} P<0.05$. (H) Growth curves of orthotopic U251/pSR (vector) and $\mathrm{U} 251 \mathrm{HIF} 1 \mathrm{KD}_{1+4}$ tumors using $\mathrm{BLI}(n=7)$. A whole brain irradiation was given on day 22. Error bars indicate SD. ${ }^{*} P<0.05$. 
A

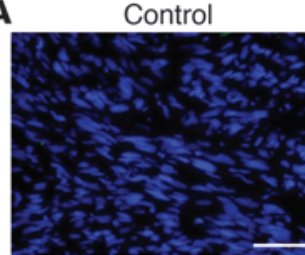

IR 2 wk

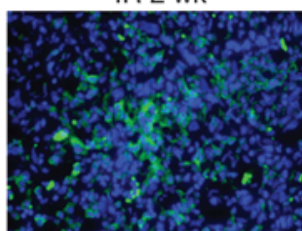

DAPI SDF-1

c

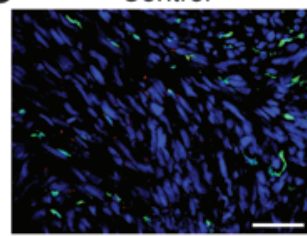

DAPI GFP pCXCR4

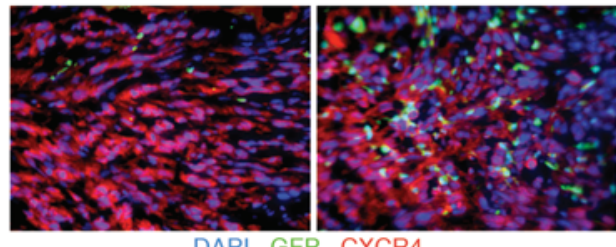

DAPI GFP CXCR4

E
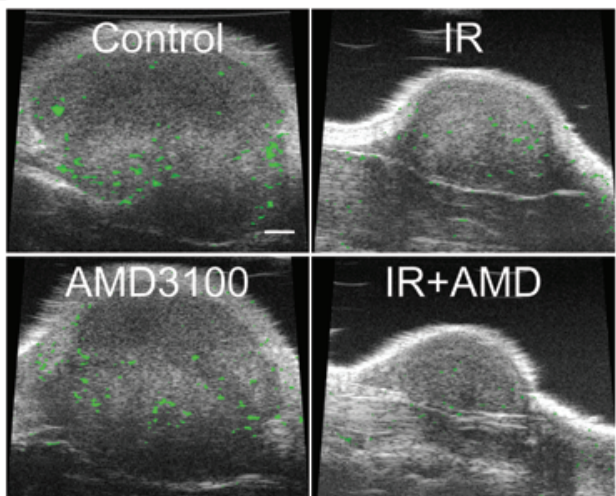

B

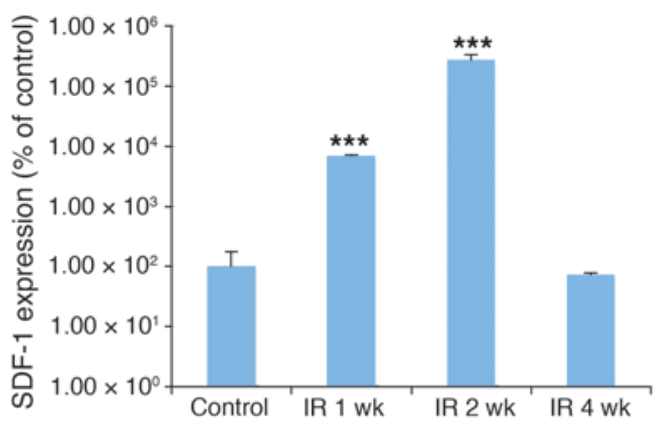

D

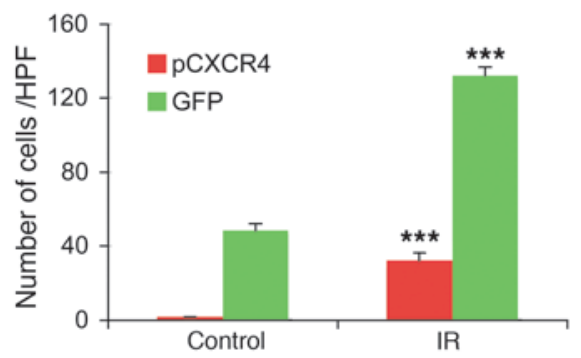

F

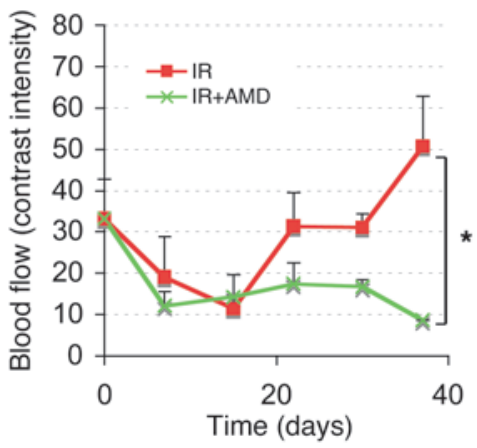

Figure 3

The interaction of SDF-1 and CXCR4 promotes tumor influx of BMDCs and restoration of tumor vasculature. (A) Irradiation promotes the expression of SDF-1 in U251 i.c. tumor. Representative image of IHC staining for SDF-1. Scale bar: $50 \mu \mathrm{m}$. (B) Quantification of SDF-1 in the irradiated tumors. Error bars indicate SEM. ${ }^{* *} P<0.001$ versus control. (C) Phosphorylation of CXCR4 on BMDCs in tumors was induced after irradiation. IHC staining for GFP-BM and pCXCR4 in U251 i.c. tumors 3 weeks after 15 Gy whole brain irradiation. Arrowheads indicate phospho-CXCR4 GFP-BM cells. Scale bar: $50 \mu \mathrm{m}$. (D) Quantification of pCXCR4 GFP-BM cells in U251 i.c. tumor after irradiation. Error bars indicate SEM. ${ }^{\star \star \star} P<0.001$. (E) AMD3100 prevents the restoration of tumor blood flow (green) after irradiation. Representative ultrasound images from U251 s.c. tumors treated with 15 Gy irradiation, AMD3100, irradiation+AMD3100, and control. Scale bar: $1 \mathrm{~mm}$. (F) Quantification of blood flow in tumor of E. Blood flow was reduced by irradiation but recovered by 3 weeks. AMD3100 plus IR prevents the recovery of tumor blood flow. Error bars indicate SD. ${ }^{\star} P<0.05$.

the growth of the unirradiated tumor was not affected by NSC134754, the response of the irradiated tumor was profoundly changed, with constant shrinkage and no regrowth over the time of the experiment (Figure $2 \mathrm{G}$ ), even after the end of the treatment with NSC-134754.
We also determined the effect of HIF-1 on the radiosensitivity of GBM by genetic means, using both brain-implanted HIF-1 $\alpha-$ knockdown (KD) U251 cells and Hif1a-KO mouse GBM cells. Increased tumor radiosensitivity was found in the HIF-1-KD tumors that depended on the KD levels of HIF-1 in i.c. U251 (Fig- 
A
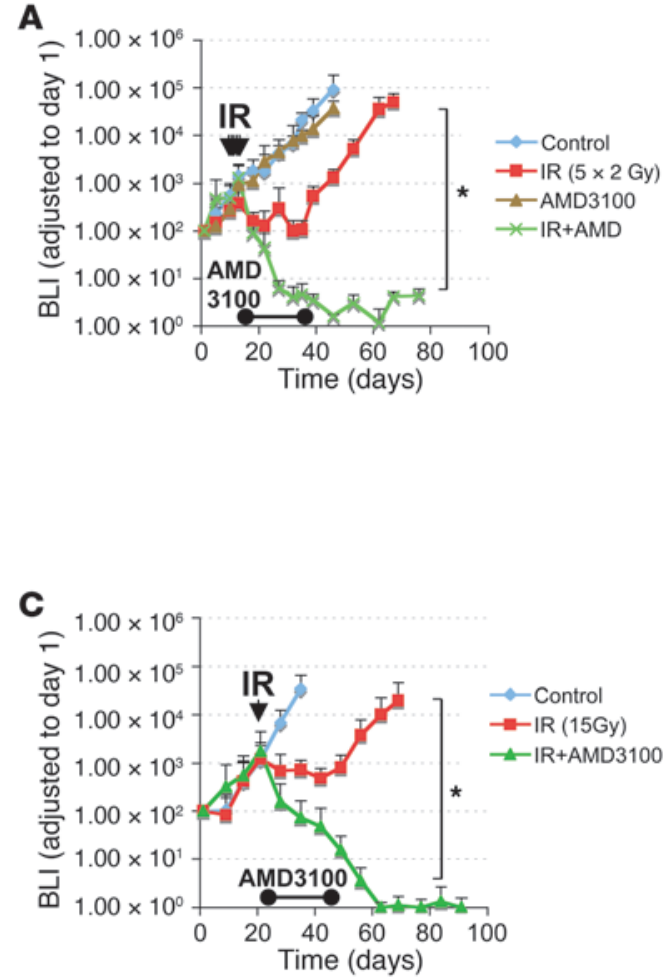

E

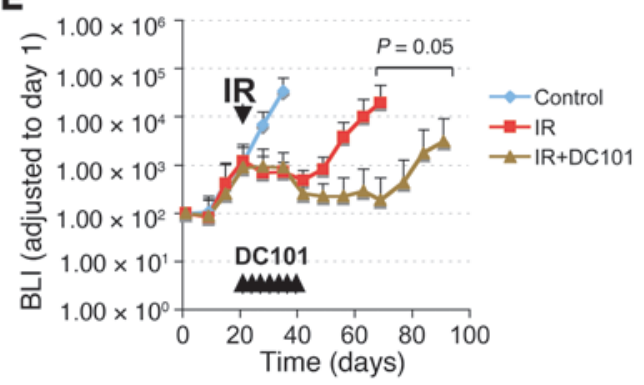

B
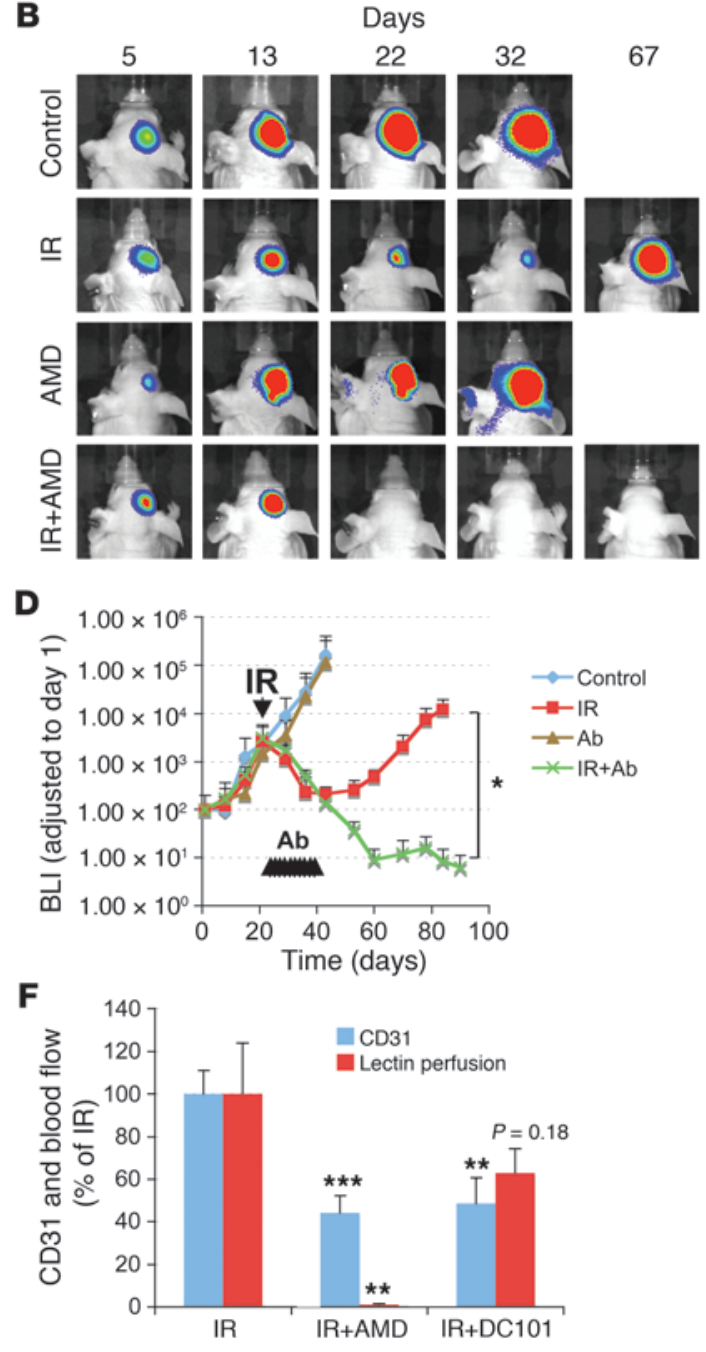

Figure 4

Therapeutic effect of blocking the interaction of SDF-1 with CXCR4 after whole brain irradiation. (A) Growth curves of i.c. U251 early tumor model after fractionated irradiation (5 daily doses of 2 Gy starting on day 11 after transplantation). ${ }^{*} P<0.05$. (B) BLI images after fractionated irradiation treated with or without AMD3100. (C and D) Growth curves of i.c. U251 advanced tumor model after a single dose of irradiation (15 Gy on day 22 after transplantation), treated with AMD3100 (21 day infusion; C, ${ }^{*} P<0.05$ ) or with neutralizing anti-CXCR4 Ab (D, $\left.{ }^{*} P<0.05\right)$, starting immediately after irradiation. (E) Growth curves of U251 i.c. tumor after 15 Gy irradiation, treated with DC101. Arrowheads indicate the treatment of DC101 (started immediately after irradiation and maintained for 21 days). (F) AMD3100 is more effective than DC101 in reducing tumor blood flow. Quantification of endothelial cells and functional vessels in U251 s.c. tumors after 15 Gy irradiation and combined with AMD3100 or DC101. Samples were taken 17 days after irradiation. Error bars indicate SEM. ${ }^{* \star} P<0.01,{ }^{* \star *} P<0.001$ versus IR.

ure $2 \mathrm{H}$ and Supplemental Figure 4, A and B). Hif-1-null murine GBM tumors were also more sensitive to irradiation (Supplemental Figure 4, C and D), resulting in longer survival of the Hif-1-KO tumor-bearing mice compared with the control tumor-bearing mice (Supplemental Figure 4E). This increase in tumor radiosensitivity of the HIF- $1 \alpha-$ KD tumors was not the result of any change in radiosensitivity or growth of the cells in vitro (data not shown). These data suggest that blocking the HIF-1 signal caused by local irradiation attenuates recruitment of $\mathrm{CD} 11 \mathrm{~b}^{+} \mathrm{BMDCs}$, thereby preventing tumor vasculogenesis and tumor regrowth.

HIF-1-induced SDF-1 activates its receptor and sustains tumor blood flow. Several investigators have shown that BMDCs are recruited to and retained in hypoxic normal tissues and in tumors by the hypoxia-dependent secretion of SDF-1, which binds to its receptor, CXCR4, on BMDCs $(7,29-32)$. Indeed, we found that SDF-1 secretion was upregulated under hypoxic conditions in vitro, and this upregulation was abolished by NSC-134754 (data not shown). In our brain-implanted GBM, SDF-1 levels in the tumors rose to a maximum level by 2 weeks after irradiation and then returned to control levels at 4 weeks (Figure $3, \mathrm{~A}$ and B). In addition, we found that local irradiation increased phosphorylation of CXCR4 in the tumor, indicating interaction of SDF-1 with CXCR4 (Figure $3, \mathrm{C}$ and D). Approximately $20 \%$ of the BMDCs recruited into the tumor after irradiation exhibited phospho-CXCR4, whereas very 
A

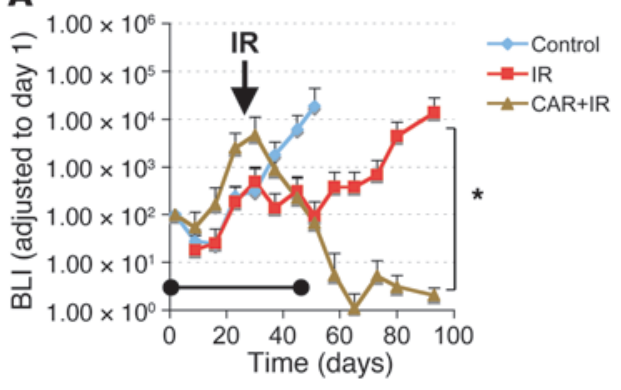

B

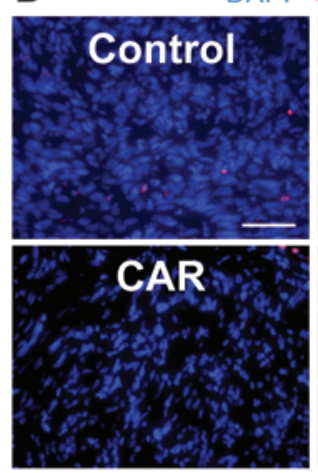

D

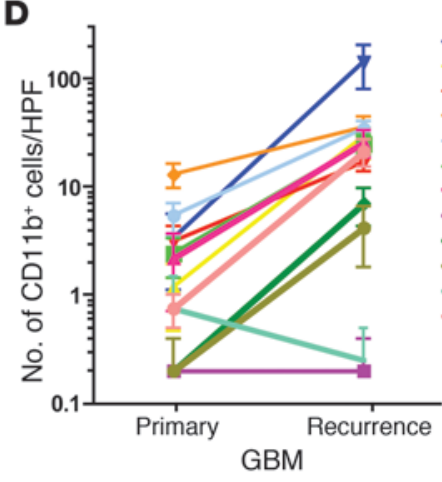

C

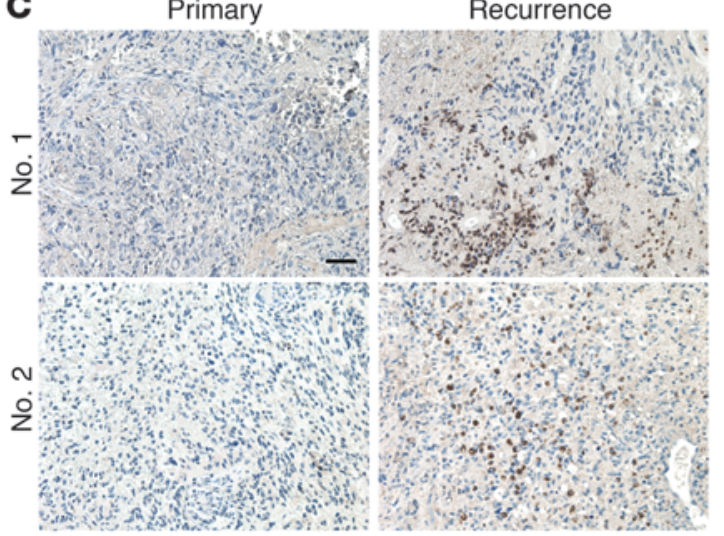

Recurrence
Figure 5

CD11 $b^{+}$cells are associated with GBM tumor recurrences in U251 i.c. tumors in mice and in patients. (A) Growth of U251 i.c. tumor assessed by BLI. Tumor-bearing mice were treated with 15 Gy whole brain irradiation, carrageenan (CAR), or the combination of irradiation and CAR. The arrow indicates irradiation, and the bar indicates the period of CAR treatment. Error bars indicate SD. ${ }^{*} P<0.05$. (B) IHC of U251 i.c. tumor of $\mathbf{A}$ for CD11b. The average of CD11b cells per HPF was 6.3 (control), 72.6 (IR), 2.6 (CAR), and 2.0 (CAR+IR). (C) IHC of GBM clinical samples staining for CD11b. (D) Significantly increased levels of CD11b+ cells in the recurrent human GBMs compared with the untreated tumors. Quantification of CD11b based on IHC with CD11b staining. Eight of twelve samples showed significant increases in CD11b+ cells in recurrent GBMs $(P<0.05)$. Error bars indicate SEM. Scale bars: $50 \mu \mathrm{m}$. low levels of phosphorylation of BMDCs were found prior to irradiation (Supplemental Figure 5A).

To determine the effect of inhibiting the interaction of SDF-1 with CXCR4 on tumor blood flow in the irradiated tumors, we used the clinically approved drug AMD3100, an inhibitor of the interaction of SDF-1 with CXCR4, utilizing the technique of ultrasound analysis with microbubbles (41) in the U251 s.c. tumor. As shown (Figure 3, E and F, and Supplemental Videos 1 and 2), AMD3100 had no effect on blood flow compared with control tumor but prevented the return of blood flow in the irradiated tumors. These data suggest that HIF-1-upregulated SDF-1 is an important factor for retention of BMDCs in the irradiated tumor and can promote the recovery of the tumor blood supply after its reduction by irradiation.

Blocking SDF-1 prevents tumor recurrence following irradiation and is significantly more effective than VEGF blockage. We next investigated the therapeutic effect of AMD3100 in blocking the interaction of SDF-1/CXCR4 stimulated by irradiation. Tumor-bearing mice were infused with AMD3100 starting immediately after irradiation. This had no significant effect on the growth of unirradiated of the tumor, as demonstrated by tumor growth and by mouse survival time (Supplemental Figure 6, A and B).

It has been reported that tumor-produced VEGF not only promotes local angiogenesis but also mobilizes endothelial and hematopoietic progenitors from the $\mathrm{BM}(42,43)$. As VEGF is also an HIF-1 target gene, we tested the inhibition of VEGF using the mouse VEGFR2-neutralizing monoclonal antibody DC101. DC101 treatment alone slightly reduced the tumor growth rate (Supplemental Figure 5B). As shown in Figure 4E, this treatment given for 3 weeks after irradiation delayed the regrowth of the tumors but was not as effective as AMD3100 (or the HIF-1 inhibitor NSC134754 ) in preventing recurrence of the irradiated tumors. We also compared the effect of AMD3100 and DC101 on tumor vascularity and blood perfusion after irradiation. Whereas both agents decreased the number of endothelial cells in the irradiated tumors, only AMD3100 produced an essentially total blockage of tumor perfusion when combined with irradiation (Figure 4F).

We further tested whether AMD3100 prevented the vasculogenesis pathway by using the model system of transplanting tumors into an irradiated normal tissue to eliminate local angiogenesis. We found 
A
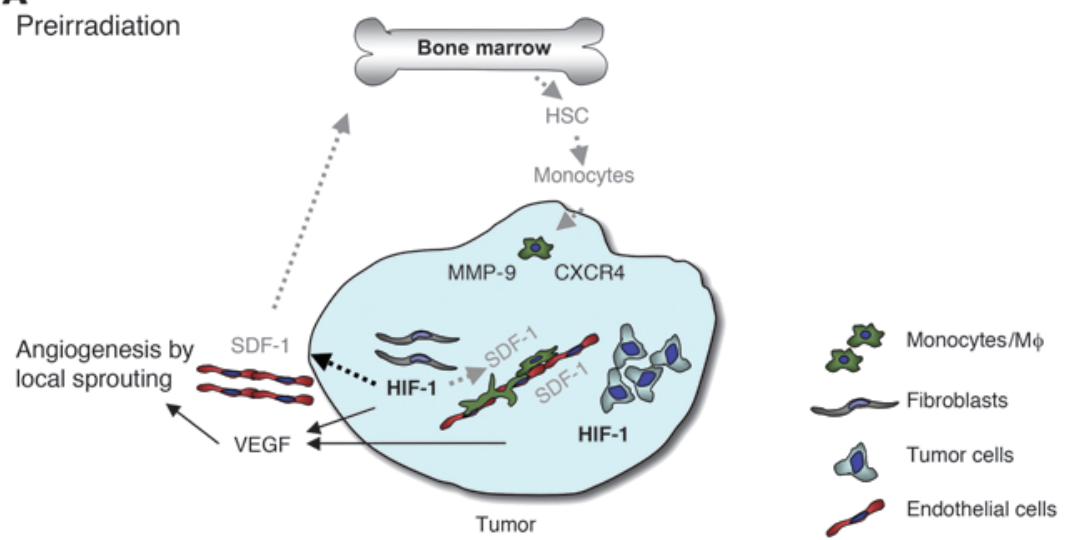

B

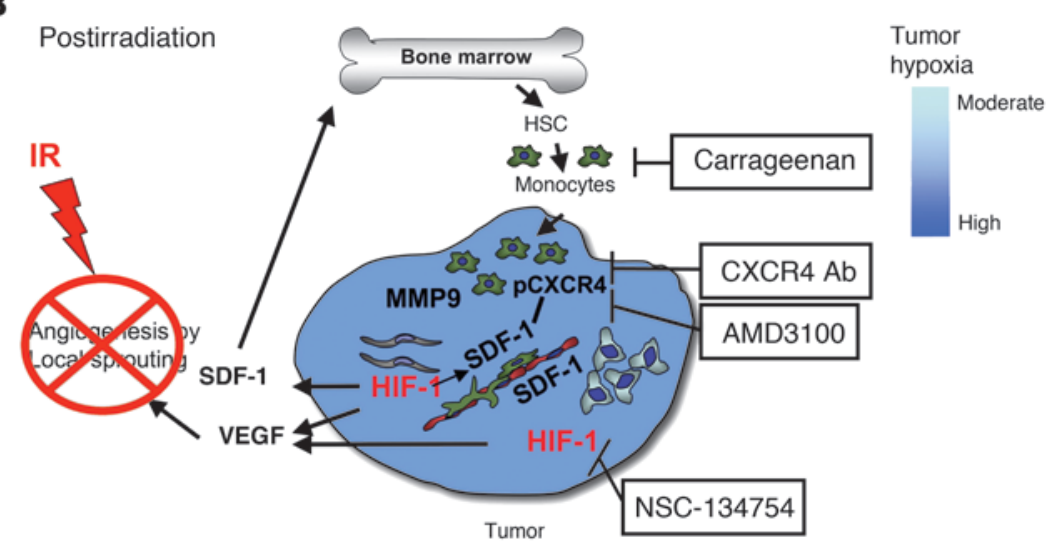

\section{Figure 6}

Model of the main contributions of BMDCs; and cytokines that promote restoration of tumor vasculature after irradiation. (A) Prior to irradiation, tumor growth is governed largely by local angiogenesis. When local angiogenesis is inhibited by irradiation, growth of the tumor vasculature (essential for recurrence of the tumor) can only occur from circulating cells, of which BMDCs are an essential component. (B) After irradiation, the tumor becomes more hypoxic, and HIF-1 is increased as the tumor attempts to regrow. This induces SDF-1 and promotes the mobilization of $C D 11 b^{+}$monocytes from the BM and retention of these BMDCs into the tumor. SDF-1 is the key factor for the influx of BMDCs, as AMD3100, an inhibitor of CXCR4/ SDF-1, and antibodies against CXCR4 block the recruitment and/or tumor retention of the BMDCs, inhibit restoration of the tumor vasculature, and prevent tumor recurrence. The various inhibitors and the points in the cycle at which they act are shown in boxes. M $\phi$, macrophages. that HIF-1 KD or treatment of tumor-bearing mice with AMD3100 totally abrogated tumor growth in the preirradiated normal tissue (Supplemental Figure 6, A and B). These data show that tumor regrowth is absolutely dependent on the vasculogenesis pathway when angiogenesis is impaired by local irradiation and that blocking the HIF-1 or SDF-1/CXCR4 signal prevents tumor recurrence.

Myelomonocytic cells are critical for tumor regrowth after irradiation. We next tested the importance of myelomonocytic cells for tumor regrowth following irradiation. To do this, we depleted the monocyte/macrophage lineage using carrageenan (44). This treatment reduced the number of $\mathrm{CD} 11 \mathrm{~b}^{+}$BMDCs in the $\mathrm{U} 251$ i.c. tumor (Figure 5B). We also found that this prevented tumor recurrence after irradiation, although depletion of myeloid cells did not reduce the growth rate of unirradiated i.c. tumor (Figure 5A and Supplemental Figure 8A). These data are consistent with previous reports suggesting involvement of myeloid lineage cells in tumor progression and vasculogenesis $(20,22,35)$.

$C D 11 b^{+}$myelomonocytic cells are increased in recurrences of buman GBM. Finally, we determined the level of CD11 $\mathrm{b}^{+}$cells in human GBM tumors both prior to therapy and when the tumors had recurred in the same patients. We observed higher levels of CD11b+ cells in the recurrent tumors compared with those in the untreated tumors in 10 of the 12 paired samples, which was statistically significant $(P<0.05)$ in 8 of the 12 pairs (Figure $5, \mathrm{C}$ and $\mathrm{D})$. CD11b $\mathrm{b}^{+}$cells were not found or were at undetectable levels in normal brain tissues, indicating that the presence of large number of infiltrating CD $11 \mathrm{~b}^{+}$ cells in recurrent tumors is stimulated by tumor-derived cytokines.

\section{Discussion}

The present series of experiments tested our hypothesis that the growth of a tumor, after radiation doses similar to those given in radiotherapy, has to rely on the vasculogenesis pathway, which is dependent on circulating cells, primarily of BM origin (Figure 6), and that inhibition of this pathway will prevent, or delay, tumor recurrence. Our results in the i.c. implanted GBM in mice support this hypothesis. We demonstrate that there is a large influx of BMDCs in the irradiated tumors as they begin to regrow after irradiation, and that 3 different ways of abrogating this influx (inhibition of HIF-1 or SDF-1/CXCR4 or depletion of monocytes/ macrophages) prevent, or substantially delay, tumor recurrence. Our data suggest that the stimulus for the influx of BMDCs is increased tumor hypoxia caused by loss of functional vasculature after irradiation. Notably, we demonstrate that hypoxia is dramatically increased in the well-vascularized U251 brain tumor as it initiates regrowth after irradiation and this coincides with increased tumor HIF-1 levels. Further, inhibition of HIF-1 or inhibition of the interaction of the HIF-1-upregulated SDF-1 with its receptor, CXCR4, after irradiation prevents both the recruitment of BMDCs into the tumors and their regrowth after irradiation.

As discussed earlier, the 2 principal ways in which a tumor can expand its vasculature as it grows is by angiogenesis involving sprouting of endothelial cells from nearby normal vessels or by vasculogenesis, which occurs by the recruitment into the tumor of circulating endothelial and other cells. Our data suggest that for the intracranial growth of the U251 tumor, the angiogenesis 
pathway is dominant, as none of the 3 methods we used to inhibit vasculogenesis affected the growth of the unirradiated control tumors (Figures 2, 4, and 5). However, once the tumor received irradiation, most of the $\mathrm{CD} 31^{+}$endothelial cells in the tumor were eradicated (Supplemental Figure 2E). Also, those in the surrounding normal vessels would be expected to be sterilized, thereby inhibiting the angiogenesis pathway. Indeed, we and others have shown that irradiation abrogates tumor angiogenesis (45). This loss of functional vasculature in the tumor combined with an inability of the normal vasculature to provide vessels is likely the reason for the large increase in tumor hypoxia we observed 2 weeks after irradiation (Figure 2, A and B) and the resulting increase in tumor HIF-1 levels (Figure 2C), thereby providing the stimulus for vasculogenesis. It has been reported that local irradiation promotes HIF-1 activation due to tumor reoxygenation and increased free radicals (28). In our i.c. tumor model, HIF-1 was activated by local irradiation, but not by the same mechanism as reported, because it occurred much later than that caused by free radical production and was more consistent with the loss of tumor vasculature. Consistent with HIF-1 being the signal for vasculogenesis, we show that both pharmacological and genetic inhibition of HIF-1 attenuated BMDC recruitment and inhibited tumor recurrence. Of note is the fact that the effect of the HIF-1 inhibitor NSC-134754 is greater than that of HIF-1 KD or KO in the tumor cells (Figure 2 and Supplemental Figure 3). The likely reason for this is that in the HIF-1-KO tumors there are also stromal cells that can express HIF-1 (24).

AMD3100, an FDA-approved orphan drug, promotes the mobilization of BMDCs from the BM niche to peripheral blood by inhibiting the interaction of SDF-1 with CXCR4 (46). As SDF-1 is also known to have an important role in retention of CXCR4 ${ }^{+}$ BMDCs in perivascular hypoxic areas of ischemic tissue (7, 29-32), we used AMD3100 to inhibit this part of the vasculogenesis pathway. Although it has been reported that SDF-1 inhibition by AMD3100 increases the therapy-induced growth delay of GBM in a mouse model by inhibiting the Erk and Akt pathways (47), this is not the mechanism of the effect we observed for 2 reasons. First, we only applied the drug after tumor irradiation, thus making any effect on tumor cell radiosensitivity unlikely. Second, we saw no effect of AMD3100 on the growth of the cells in vitro with or without irradiation. However, the effect of this drug in blocking BMDC influx and tumor regrowth was dramatic after either single or fractionated doses of irradiation. An important feature of our data is that although we completed the drug infusion before the tumors were eradicated, the tumors continued to shrink and did not recur even long after the end of the infusion.

The BMDC accumulation in the U251 tumors following irradiation was composed largely of $\mathrm{CD} 11 \mathrm{~b}^{+}$monocytes. These cells have been shown to be increased in the BM, spleens, and peripheral blood of tumor-bearing mice and in the peripheral blood of cancer patients $(48,49)$. There is strong clinical evidence that infiltration levels of macrophages that are of myeloid cell lineage correlate with poor prognosis in breast, prostate, ovarian, and cervical cancers (50). In our tumor model, irradiation significantly increased the number of CD11 $\mathrm{b}^{+}$myeloid cells in the tumor. These cells are highly proangiogenic, as we and others have reported, suggesting that they are attractive targets for enhancing tumor response to irradiation $(20,22)$. In addition, it has been reported that $\mathrm{CD} 11 \mathrm{~b}^{+} \mathrm{Gr}-1^{+}$myeloid cells mediate tumor refractoriness to anti-VEGF treatment (51). A significant finding in the irradiated tumors was that approximately $50 \%$ of the CD $11 \mathrm{~b}^{+}$infiltrating monocytes also expressed the angiopoietin receptor Tie2, defining them as Tie2-expressing monocytes (TEMs). These have been shown to be highly related to tumor-associated macrophages (TAMs) and to express a gene signature associated with active remodeling and angiogenesis (52). Significantly, these cells were undetectable in the unirradiated U251 tumors. Although it is possible that CD11b- BMDCs contribute to the vasculogenesis after irradiation, we found no evidence for the involvement of EPCs. However, in other studies, we have found that antibody neutralization of CD11b had a significant radiosensitization effect on transplanted tumors, whereas depletion of $\mathrm{Gr}-1^{+}$cells did not show the effect (G.O. Ahn et al., unpublished observations). This implicates $\mathrm{CD}_{11} \mathrm{~b}^{+} \mathrm{Gr}-1^{-}$cells as the important proangiogenic population, a conclusion similar to that of F. Pucci et al. (52).

Anti-VEGF treatment combined with irradiation is an emerging strategy currently being tested in the treatment of GBM. However, recent reports of preclinical data indicate that the benefits are transitory, followed by rapid regrowth and increased tumor aggressiveness $(53,54)$. Our data also show that inhibiting the VEGF pathway through blockage of VEGFR2 by DC101 produces an increase in growth delay by irradiation. However, the effect was transitory, and the tumors regrew following the cessation of the treatment (Figure 4E). In addition, VEGF blockade was less effective than AMD3100 treatment in inhibiting tumor perfusion after irradiation (Figure 4F). Thus, blocking angiogenesis is inferior to inhibiting the vasculogenesis pathway in preventing both the postirradiation return of tumor perfusion and tumor recurrences.

In summary, our data suggest that the novel strategy of inhibiting the vasculogenesis pathway after local tumor irradiation has the potential to improve the control of human GBM by radiotherapy. In essence, this strategy abolishes the need to kill all the tumor cells, replacing it with the need only to kill the endothelial cells in and around the tumor, which will require significantly lower radiation doses than those needed to sterilize all the tumor cells.

\section{Methods}

Cell culture and retroviral transduction. U251 and U87MG cells were obtained from R.K. Puri (FDA, Bethesda, Maryland) and maintained in DMEM with 10\% FBS (Gibco, Invitrogen). Murine GBM Hif1a wild-type and KO cell lines were obtained from G. Bergers (UCSF, San Francisco, California) and maintained in DMEM with $10 \%$ FBS.

The retrovirus-mediated RNA interference study was performed with the pSuper RNA interference system (Oligoengine). HIF-1 $\alpha$ target sequences were as follows: HIF1KD 1 , 5'-GGAAATGAGAGAAATGCTTAC-3'; HIF1KD 2 , 5'-GCTGGAGACACAATCATATCT-3'; HIF1KD 3 , 5'-GCTTTGAGTCAAAGAACTACA-3'; HIF1KD 4 , 5'-GAGGAAGTACCATTATAT-3'. HIF1KD 4 was obtained from D. Chan (Stanford University, Palo Alto, California). For retroviral packaging, 293T cells were cotransfected with pVPack-VSV-G, pVPack-GP (Stratagene), and the HIF-1 $\alpha-$ KD or pSUPERretro (pSR) control constructs, and viral supernatant was harvested after 48 hours. The transduction of the firefly luciferase gene was performed with the pFB-Luciferase retrovirus system (Stratagene). For infections, cells were incubated with viral supernatant in the presence of $1 \mu \mathrm{g} / \mathrm{ml}$ DEAEdextran, and after 48 hours, infected cells were selected with puromycin $(1 \mu \mathrm{g} / \mathrm{ml})$ for 72 hours.

BM transplantation. All animal procedures were approved by Stanford University's Administrative Panel on Laboratory Animal Care (APLAC). Athymic $n u / n u$ nude mice were purchased from Charles River Laboratories. GFP nude mice were obtained from AntiCancer Inc. Mice were 
maintained in a germ-free environment and had access to food and water ad libitum. BM was harvested from 6- to 12-week-old GFP nude mice by flushing the femurs and tibias with $2 \%$ FBS in Hank's balanced salt solution (Invitrogen). Cells $\left(>2 \times 10^{6}\right)$ were injected i.v. through the tail vein of lethally irradiated $(8.5 \mathrm{~Gy})$ recipient mice. Tumor cell implantation was performed i.c. or s.c. 4 weeks later.

Orthotopic i.c. tumor implantation and irradiation. For i.c. implantation of U251, U87, or murine GBM cells, 6- to 12-week-old nude mice were implanted i.c. as described previously (55) with $5 \mu \mathrm{l}$ of $1 \times 10^{6} \mathrm{U} 251 / \mathrm{pFB}-$ Luc, U251/pFB-LucHIF1KD, U251/5HRE-Luc, U87/pFB-Luc or $1 \times 10^{5}$ murine pFB-Luc GBM HIF1 WT or KO cells. Anesthetized mice were placed in individual lead boxes with a cut-out through which the whole brain protruded for irradiation but that shielded the mouth and throat cavity. All treatment experiments were repeated 2-3 times.

For the s.c. tumor inoculation, U251 cells were inoculated at $1 \times 10^{7}$ cells/ mouse on the back of the mouse approximately $1 \mathrm{~cm}$ proximal to the base of the tail. Unanesthetized mice were placed in lead jigs through which the established tumors (approximately $200 \mathrm{~mm}^{3}$ in volume) protruded for irradiation.

Irradiation was performed with a Phillips X-ray unit operated at $200 \mathrm{kVp}$ with a dose rate of $1.21 \mathrm{~Gy} / \mathrm{min}(20 \mathrm{~m} \AA$ with added filtration of $0.5 \mathrm{~mm}$ copper, distance from X-ray source to the target of $31 \mathrm{~cm}$, and a half value layer of $1.3 \mathrm{~mm}$ copper). Tumor volume $(V)$ was calculated using the formula for a spheroid: $V=0.5 \times$ width $\times$ length $\times$ depth.

In vivo real-time optical imaging. Mice were anesthetized and injected i.p. with $150 \mathrm{mg} / \mathrm{kg}$ D-luciferin (Caliper). Imaging was completed between 5 and 30 minutes after injection with the IVIS system (Caliper) coupled to Living Image acquisition and analysis software. For bioluminescence index (BLI) plots, photon flux was calculated for each mouse by using a rectangular region of interest encompassing the head of the mouse in a prone position. This value was scaled to a comparable background value (from a luciferin-injected mouse with no tumor cells) and then normalized to the value obtained immediately after xenografting (day 0 ), so that all mice had an arbitrary starting BLI signal of 100. Error bars shown in figures indicate SD.

Ultrasound analysis. Ultrasound imaging was performed using a dedicated small-animal high-resolution imaging unit (Vevo 770; VisualSonics). Mice were anesthetized with isoflurane and maintained in a supine position. The animals' body temperature was maintained with a heated platform and an auxiliary heat lamp. A tail vein cannulation was performed using a 27-gauge butterfly needle apparatus (VisualSonics), through which all contrast agents were injected. To measure microvascular perfusion, a $50-\mu$ l bolus $\left(1 \times 10^{8}\right.$ microbubbles $)$ of Non-Targeted MicroMarker Contrast Agent was injected through the tail vein cannula. The first-pass bolus wash-in was viewed and used to quantify blood flow. The change in intensity from baseline to plateau gave an indication of relative blood volume, and the slope of the curve represented the relative blood flow.

Drug treatment. We obtained DC101 hybridoma from C.J. Kuo (Stanford University) and NSC-134754 from Developmental Therapeutics Program (NCI/NIH, Bethesda, Maryland). Nude mice injected i.c. with U251 cells were randomized into 4 cohorts $(n=5-7)$ for treatment with inhibitors alone (NSC-134754, AMD3100, or DC101), irradiation alone, inhibitors combined with irradiation, or control. Treatment with all the inhibitors was started immediately after irradiation and maintained for 3 weeks. The doses, sources, and routes of drug administration were as follows: NSC134754 (Developmental Therapeutics Program, NCI/NIH): 5 mg/kg dose, injected i.p. daily; AMD3100 (Sigma-Aldrich): $5 \mathrm{mg} / \mathrm{kg}$, continuous infusion by osmotic pump (Durect); neutralizing anti-CXCR4 antibody (Torrey Pines Biolabs): $0.4 \mathrm{mg} / \mathrm{kg}$, injected i.p. daily; DC101: $40 \mathrm{mg} / \mathrm{kg}$, purified from hybridoma supernatant as described previously (56), injected i.p. every 3 days. To deplete the monocyte/macrophage lineage, carrageenan
( $2 \mathrm{mg} /$ mouse) was i.p. injected 7,3 , and 1 day prior to tumor inoculation and continued twice per week for the period indicated in Figure 4.

Body weight of the mice was monitored during all the treatments. No significant losses in body weight (less than $10 \%$ ) were observed.

TaqMan qPCR. Total RNA was extracted from mouse brain including U251 i.c. tumor by TRIzol (Invitrogen). After reverse transcription, cDNA was then subjected to qPCR with $g f p$-specific TaqMan probe and primer set (probe: 5'-6FAM-CCAGTCCGCCCTGAGCAAAGACC-MGB-3'; forward primer: 5'-CTGCTGCCCGACAACCAC-3'; reverse primer: 5'-TCACGAACTCCAGCAGGAC-3').

$M R I$. High-resolution MR images were acquired on a 7.0-T/31-cm animal scanner system (Varian). The imaging protocol consisted of scout imaging in 2 planes, followed by a spin-echo sequence $(\mathrm{TR}=2,500 \mathrm{~ms}, \mathrm{TE}=45$ $\mathrm{ms}, \mathrm{NEX}=4$, matrix $=256 \times 256, \mathrm{FOV}=30 \mathrm{~mm}$, slice thickness $=1 \mathrm{~mm}$, gap $=0 \mathrm{~mm}$ ). During imaging, animals were under isoflurane anesthesia and monitored for respiration and heart rate; the temperature was kept at $37^{\circ} \mathrm{C}$. Images were analyzed by OsiriX DICOM viewer.

Tissue preparation. When the luciferase signal reached approximately the same as that at irradiation, we performed cardiac perfusion in asphyxiated tumor-bearing mice with PBS, followed by $4 \%$ paraformaldehyde in PBS. To determine the hypoxia level, we injected pimonidazole hydrochloride 90 minutes prior to tissue harvest. For visualization of the functional tumor vessels, biotinylated tomato lectin was administered via tail vein injection before performing cardiac perfusion. Tumors were removed, embedded in optimal cutting temperature (OCT) compound (Sakura Finetek), and frozen at $-80^{\circ} \mathrm{C}$ until cryosectioning and immunostaining.

IHC. Frozen sections were used for immunohistochemical analysis. Tumor endothelial cells were visualized by staining with a 1:500 dilution of rat anti-mouse CD31 antibody (BD Biosciences). BMDC populations were analyzed by staining with 1:500 anti-mouse CD11b, F4/80, CD45, Gr-1 (BD Biosciences), VEGFR1 (Abcam), VEGFR2, Tie-2 (eBioscience) antibodies or by detecting GFP in GFP-BM-transplanted mice. Phosphorylation of CXCR4 was visualized with anti-phosphorylated CXCR4 antibody obtained from J.B. Rubin (Washington University in St. Louis, Missouri). SDF-1 was detected with anti-SDF-1 antibody (Novus Biologicals). Hypoxic areas were detected with FITC-conjugated antiHypoxyprobe-1 antibody (Natural Pharmacia International). CD11b staining was performed on paraffin-embedded human GBM specimens with anti-human CD11b antibody (Abcam). For fluorescence visualization of antibody reactions, primary antibodies were detected using secondary antibodies labeled with the fluorochromes Alexa Fluor 488, Alexa Fluor 555, or Alexa Fluor 594 or by streptavidin labeled with Alexa Fluor 488 or Alexa Fluor 555 (1:1,000; Molecular Probes), while nuclei were detected with DAPI. Photomicrographs were taken with a Leica DM6000B microscope using Plan $10 \times / 0.30,20 \times / 0.50$, and $40 \times / 0.85$ objective lenses with $10 \times / 25$ eyepieces.

Quantification of expression and colocalization. Fluorescence images of tumor sections were acquired with a QImaging digital camera and Imaging Pro Plus software (MediaCybernetics). The average of signal intensity for $\mathrm{CD} 31$, lectin, or pimonidazole staining was measured by the software from 4 or 5 random fields using $\times 20$ objective with $\times 10$ eyepieces of the fluorescence microscope. Quantitative analysis for GFP-BM, CD11b, and phospho-CXCR4-positive cells was performed by determining the number of cells in 5 randomly photographed fields using a $\times 40$ objective with $\times 10$ eyepieces of the fluorescence microscope. Quantification was performed in 4-6 animals per group. Error bars shown in the figures indicate SEM.

Histological assessment. Human GBM specimens of primary tumor and recurrences after radiation therapy were obtained from biopsy or surgical resection by the Department of Neurosurgery at Stanford Hospital with informed consent according to US federal law and are exempt from 
consideration by the Stanford Administrative Panel on Human Subjects in Medical Research.

Immunoblotting. U251 HIF1KD cells were cultured under hypoxic conditions $\left(0.5 \%\right.$ or $2 \% \mathrm{O}_{2}$ for 48 hours). Cell extracts were prepared in an Invivo $_{2} 400$ Hypoxia Workstation (Ruskin) by gently scraping off cells in PBS followed by extraction in $8 \mathrm{M}$ urea buffer containing $1 \times$ Complete Mini protease inhibitors (Roche). Western blot analysis was performed with $30 \mu \mathrm{g}$ protein with anti-human HIF-1 $\alpha$ monoclonal antibody $(1: 1,000)$ or an anti-GAPDH antibody $(1: 5,000)$ as loading control.

Statistics. Statistical analyses were performed by the 2-tailed Student's $t$ test or 1-way ANOVA to determine statistical significance. $P$ values (exact significance) of less than 0.05 were considered statistically significant. Kaplan-Meier curves and the log-rank test were used to compare survival times among the groups. All calculations were performed using Prism 4 (GraphPad).

1. Souhami L, et al. Randomized comparison of stereotactic radiosurgery followed by conventional radiotherapy with carmustine to conventional radiotherapy with carmustine for patients with glioblastoma multiforme: report of Radiation Therapy Oncology Group 93-05 protocol. Int J Radiat Oncol Biol Phys. 2004;60(3):853-860.

2. Hochberg FH, Pruitt A. Assumptions in the radiotherapy of glioblastoma. Neurology. 1980;30(9):907-911.

3. Liang BC, Thornton AF Jr, Sandler HM, Greenberg HS. Malignant astrocytomas: focal tumor recurrence after focal external beam radiation therapy. J Neurosurg. 1991;75(4):559-563.

4. Sneed PK, et al. Patterns of recurrence of glioblastoma multiforme after external irradiation followed by implant boost. Int J Radiat Oncol Biol Phys. 1994;29(4):719-727.

5. Garcia-Barros M, et al. Tumor response to radiotherapy regulated by endothelial cell apoptosis. Science. 2003;300(5622):1155-1159.

6. Ogawa K, Boucher Y, Kashiwagi S, Fukumura D, Chen D, Gerweck LE. Influence of tumor cell and stroma sensitivity on tumor response to radiation. Cancer Res. 2007;67(9):4016-4021.

7. Ceradini DJ, et al. Progenitor cell trafficking is regulated by hypoxic gradients through HIF-1 induction of SDF-1. Nat Med. 2004;10(8):858-864.

8. Takahashi $\mathrm{T}$, et al. Ischemia- and cytokine-induced mobilization of bone marrow-derived endothelial progenitor cells for neovascularization. Nat Med. 1999;5(4):434-438.

9. Rafii S, Lyden D. Therapeutic stem and progenitor cell transplantation for organ vascularization and regeneration. Nat Med. 2003;9(6):702-712.

10. Orlic D, et al. Bone marrow cells regenerate infarcted myocardium. Nature. 2001;410(6829):701-705.

11. Coussens LM, Werb Z. Inflammation and cancer. Nature. 2002;420(6917):860-867.

12. Condeelis J, Pollard JW. Macrophages: obligate partners for tumor cell migration, invasion, and metastasis. Cell. 2006;124(2):263-266.

13. Erler JT, et al. Hypoxia-induced lysyl oxidase is a critical mediator of bone marrow cell recruitment to form the premetastatic niche. Cancer Cell. 2009; 15(1):35-44.

14. Lyden D, et al. Id1 and Id3 are required for neurogenesis, angiogenesis and vascularization of tumour xenografts. Nature. 1999;401(6754):670-677.

15. Lyden D, et al. Impaired recruitment of bone-marrow-derived endothelial and hematopoietic precursor cells blocks tumor angiogenesis and growth. Nat Med. 2001;7(11):1194-1201.

16. Gothert JR, et al. Genetically tagging endothelial cells in vivo: bone marrow-derived cells do not contribute to tumor endothelium. Blood. 2004; 104(6):1769-1777.

17. De Palma M, Venneri MA, Roca C, Naldini L. Targeting exogenous genes to tumor angiogenesis by transplantation of genetically modified hematopoietic stem cells. Nat Med. 2003;9(6):789-795.

18. Moldovan NI, Goldschmidt-Clermont PJ, ParkerThornburg J, Shapiro SD, Kolattukudy PE. Contribution of monocytes/macrophages to compensatory neovascularization: the drilling of metalloelastase-positive tunnels in ischemic myocardium. Circ Res. 2000;87(5):378-384.

19. Capoccia BJ, Shepherd RM, Link DC. G-CSF and AMD3100 mobilize monocytes into the blood that stimulate angiogenesis in vivo through a paracrine mechanism. Blood. 2006;108(7):2438-2445.

20. Bailey AS, et al. Myeloid lineage progenitors give rise to vascular endothelium. Proc Natl Acad Sci U S A. 2006;103(35):13156-13161.

21. Ruzinova MB, et al. Effect of angiogenesis inhibition by Id loss and the contribution of bonemarrow-derived endothelial cells in spontaneous murine tumors. Cancer Cell. 2003;4(4):277-289.

22. Ahn GO, Brown JM. Matrix metalloproteinase-9 is required for tumor vasculogenesis but not for angiogenesis: role of bone marrow-derived myelomonocytic cells. Cancer Cell. 2008;13(3):193-205.

23. Bertout JA, Patel SA, Simon MC. The impact of O2 availability on human cancer. Nat Rev Cancer. 2008;8(12):967-975.

24. Du R, et al. HIF1alpha induces the recruitment of bone marrow-derived vascular modulatory cells to regulate tumor angiogenesis and invasion. Cancer Cell. 2008;13(3):206-220.

25. Sasai K, Brown JM. Discrepancies between measured changes of radiobiological hypoxic fraction and oxygen tension monitoring using two assay systems. Int J Radiat Oncol Biol Phys. 1994;30(2):355-361.

26. Zips D, et al. Impact of the tumour bed effect on microenvironment, radiobiological hypoxia and the outcome of fractionated radiotherapy of human FaDu squamous-cell carcinoma growing in the nude mouse. Int J Radiat Biol. 2001;77(12):1185-1193.

27. Rofstad EK, Mathiesen B, Henriksen K, Kindem $\mathrm{K}$, Galappathi $\mathrm{K}$. The tumor bed effect: increased metastatic dissemination from hypoxia-induced up-regulation of metastasis-promoting gene products. Cancer Res. 2005;65(6):2387-2396.

28. Moeller BJ, Cao Y, Li CY, Dewhirst MW. Radiation activates HIF-1 to regulate vascular radiosensitivity in tumors: role of reoxygenation, free radicals, and stress granules. Cancer Cell. 2004;5(5):429-441.

29. Wang Y, Haider H, Ahmad N, Zhang D, Ashraf M. Evidence for ischemia induced host-derived bone marrow cell mobilization into cardiac allografts. J Mol Cell Cardiol. 2006;41(3):478-487.

30. Jin DK, et al. Cytokine-mediated deployment of SDF-1 induces revascularization through recruitment of CXCR4+ hemangiocytes. Nat Med. 2006; 12(5):557-567.

31. Aghi M, Cohen KS, Klein RJ, Scadden DT, Chiocca EA. Tumor stromal-derived factor-1 recruits vascu- ar progenitors to mitotic neovasculature, where microenvironment influences their differentiated phenotypes. Cancer Res. 2006;66(18):9054-9064.

32. Grunewald $M$, et al. VEGF-induced adult neovascularization: recruitment, retention, and role of accessory cells. Cell. 2006;124(1):175-189.

33. Gerlach LO, Skerlj RT, Bridger GJ, Schwartz TW. Molecular interactions of cyclam and bicyclam nonpeptide antagonists with the CXCR4 chemokine receptor. J Biol Chem. 2001;276(17):14153-14160.

34. Yang M, Reynoso J, Jiang P, Li L, Moossa AR, Hoffman RM. Transgenic nude mouse with ubiquitous green fluorescent protein expression as a host for human tumors. Cancer Res. 2004;64(23):8651-8656.

35. De Palma M, et al. Tie2 identifies a hematopoietic lineage of proangiogenic monocytes required for tumor vessel formation and a mesenchymal population of pericyte progenitors. Cancer Cell. 2005;8(3):211-226.

36. Jodele $\mathrm{S}$, et al. The contribution of bone marrowderived cells to the tumor vasculature in neuroblastoma is matrix metalloproteinase-9 dependent. Cancer Res. 2005;65(8):3200-3208.

37. Song S, Ewald AJ, Stallcup W, Werb Z, Bergers G. PDGFRbeta ${ }^{+}$perivascular progenitor cells in tumours regulate pericyte differentiation and vascular survival. Nat Cell Biol. 2005;7(9):870-879.

38. Bussink J, Kaanders JH, Rijken PF, Raleigh JA, Van der Kogel AJ. Changes in blood perfusion and hypoxia after irradiation of a human squamous cell carcinoma xenograft tumor line. Radiat Res. 2000; 153(4):398-404.

39. Harada H, Kizaka-Kondoh S, Hiraoka M. Optical imaging of tumor hypoxia and evaluation of efficacy of a hypoxia-targeting drug in living animals. Mol Imaging. 2005;4(3):182-193.

40. Chau NM, et al. Identification of novel small molecule inhibitors of hypoxia-inducible factor- 1 that differentially block hypoxia-inducible factor-1 activity and hypoxia-inducible factor-1alpha induction in response to hypoxic stress and growth factors. Cancer Res. 2005;65(11):4918-4928.

41. Palmowski M, et al. Vessel fractions in tumor xenografts depicted by flow- or contrast-sensitive threedimensional high-frequency Doppler ultrasound respond differently to antiangiogenic treatment. Cancer Res. 2008;68(17):7042-7049.

42. Heissig B, et al. Recruitment of stem and progenitor cells from the bone marrow niche requires MMP-9 mediated release of kit-ligand. Cell. 2002; 109(5):625-637.

43. Hattori K, et al. Plasma elevation of stromal cellderived factor- 1 induces mobilization of mature and immature hematopoietic progenitor and stem cells. Blood. 2001;97(11):3354-3360.

44. Ishizaka S, Kuriyama S, Tsujii T. In vivo depletion of macrophages by desulfated iota-carrageenan in mice. J Immunol Methods. 1989;124(1):17-24.

45. Prionas SD, Kowalski J, Fajardo LF, Kaplan I, Kwan 
$\mathrm{HH}$, Allison AC. Effects of X irradiation on angiogenesis. Radiat Res. 1990;124(1):43-49.

46. Broxmeyer HE, et al. Rapid mobilization of murine and human hematopoietic stem and progenitor cells with AMD3100, a CXCR4 antagonist. J Exp Med. 2005;201(8):1307-1318.

47. Rubin JB, et al. A small-molecule antagonist of CXCR4 inhibits intracranial growth of primary brain tumors. Proc Natl Acad Sci U S A. 2003; 100(23):13513-13518.

48. Bronte $\mathrm{V}$, et al. Identification of a CD $11 \mathrm{~b}(+) / \mathrm{Gr}-$ $1(+) / \mathrm{CD} 31(+)$ myeloid progenitor capable of activating or suppressing CD8(+) T cells. Blood. 2000; 96(12):3838-3846.

49. Almand B, et al. Increased production of immature myeloid cells in cancer patients: a mechanism of immunosuppression in cancer. J Immunol. 2001. 166(1):678-689.

50. Pollard JW. Tumour-educated macrophages promote tumour progression and metastasis. Nat Rev Cancer. 2004;4(1):71-78.

51. Shojaei F, et al. Tumor refractoriness to anti-VEGF treatment is mediated by CD $11 \mathrm{~b}(+) \mathrm{Gr} 1(+)$ myeloid cells. Nat Biotechnol. 2007;25(8):911-920.

52. Pucci F, et al. A distinguishing gene signature shared by tumor-infiltrating Tie2-expressing monocytes, blood "resident" monocytes, and embryonic macrophages suggests common functions and developmental relationships. Blood. 2009;114(4):901-914.

53. Bergers G, Hanahan D. Modes of resistance to anti-angiogenic therapy. Nat Rev Cancer. 2008; 8(8):592-603.

54. Paez-Ribes M, et al. Antiangiogenic therapy elicits malignant progression of tumors to increased local invasion and distant metastasis. Cancer Cell. 2009; 15(3):220-231.

55. Kawakami K, Kioi M, Liu Q, Kawakami M, Puri RK. Evidence that IL-13R alpha2 chain in human glioma cells is responsible for the antitumor activity mediated by receptor-directed cytotoxin therapy. J Immunother. 2005;28(3):193-202.

56. Rockwell P, Neufeld G, Glassman A, Caron D, Goldstein NI. In vitro neutralization of vascular endothelial growth factor activation of Flk-1 by a monoclonal antibody. Mol Cell Differ. 1995;3(1):91-109. 